\title{
RAIZ QUADRADA DE OPERADORES AUTO-ADJUNTOS
}

Ester Cristina Marquezani Rocha ${ }^{1}$; Fernando Pereira de Souza ${ }^{2}$

'Aluna do Curso de Matemática - CPTL/UFMS, bolsista do Grupo PET - Matemática/CPTL/UFMS. ${ }^{2}$ Professor do Curso de Matemática - CPTL/UFMS. E-mail: estercmrocha@gmail.com

\section{RESUMO}

Inicialmente estudamos definições e propriedades relacionadas a Espaços Vetoriais e Operadores em espaços finitos. No estudo de operadores lineares, notamos que existem características particulares quando o operador linear é Auto-adjunto, mostramos que operadores lineares Autoadjuntos possuem apenas autovalores reais e que os autovetores associados a autovalores distintos são ortogonais, além disso, obtemos uma base ortogonal para o espaço vetorial finito onde este operador está definido. Outro resultado importante é o fato de existir a Raiz Quadrada Positiva Semidefinida de um Operador Auto-adjunto, tratada neste trabalho, esta Raiz Quadrada será um Operador que poderá ser encontrado através da raiz quadrada dos Autovalores.

Palavras-chave: Espaços Vetoriais, Operadores Auto-adjuntos, Decomposição Primária, Autovalores, Raiz Quadrada.

\section{INTRODUÇÃO}

Espaços Vetoriais em geral abrangem uma enorme gama de conjuntos, finitos e infinitos, focaremos o estudo nos espaços de dimensão finita, que serão chamados de espaços vetoriais Euclidianos. Nestes espaços trabalharemos os Operadores Auto-Adjuntos, que possuem características interessantes.

Primeiro mostraremos que Operadores Auto-adjuntos possuem apenas autovalores reais, mesmo trabalhando em espaços vetoriais complexos. Outro resultado importante que estudamos é o fato que autovetores associados a autovalores distintos são ortogonais, e, portanto, são linearmente independentes, este resultado nos encaminha para encontrar uma base ortogonal para o espaço vetorial.

Fizemos um estudo minucioso sobre decomposição primária e a forma canônica de Jordan, estes resultados nos auxiliam para mostrar que os autovetores de um Operador Auto-adjunto formam uma base ortogonal e, portanto a matriz da base ortogonal do operador em relação a esta é diagonal.

A base ortogonal $\mathcal{B}$, formada por autovetores nos permite definir um operador tal que $P^{2}=T$, dizemos então que $P$ é a Raiz Quadrada de $T$. Neste trabalho detalhamos a forma de encontrar a Raiz de um Operador Auto-adjunto. 


\section{METODOLOGIA}

O trabalho foi desenvolvido com base em uma pesquisa teórica e discussão do tema com o orientador através de seminários, aprofundando conhecimentos em tópicos de álgebra linear que não foram abordados ao longo do curso. O assunto abordado faz parte das atividades do programa PET Matemática - UMFS/CPTL. O trabalho incluiu uma etapa de leitura, resoluções de exercícios, desenvolvimento das atividades propostas e a tabulação dos resultados obtidos. O estudo e as atividades desenvolvidas foram avaliados através da apresentação de seminários de discussão.

\section{RESULTADOS}

Nosso estudo se baseia em espaços vetoriais de dimensão finita e operadores lineares definidos nestes espaços, começamos aqui enunciando alguns resultados preliminares.

Definição 1: Um conjunto não vazio $X$ é um espaço vetorial sobre (um corpo) $\mathbb{K}$ se em seus elementos, denominados vetores, estiverem definidas as seguintes duas operações:

(A) cada par $x, y$ de vetores de $X$ corresponde um vetor $x+y \in X$, chamado de soma de $x$ e $y$, de modo que:

(A1) $x+y=y+x, \quad \forall x, y \in X$ (propriedade comutativa),

(A2) $(x+y)+z=x+(y+z), \forall x, y, z \in X$ (propriedade associativa),

(A3) exista em $X$ um vetor, denominado vetor nulo e denotado por 0 , tal que $0+x=x$, $\forall x \in X$,

(A4) a cada vetor $x \in X$ exista um vetor em $X$, denotado por $-x$, tal que $x+(-x)=0$,

(M) A cada par $\alpha \in \mathbb{K}$ e $x \in X$, corresponde um vetor $\alpha \cdot x \in X$, denominado produto por escalar de $\alpha$ por $x$ de modo que:

(M1) $(\alpha \beta) \cdot x=\alpha(\beta \cdot x), \forall \alpha, \beta \in \mathbb{K}$ e $\forall x \in X$ (propriedade associativa),

(M2) $1 \cdot x=x, \forall x \in X$ (onde 1 é o elemento identidade de $\mathbb{K}$ ),

Além disso, vamos impor que as operações dadas em $(A)$ e $(M)$ se distribuam, isto é, que valham as seguintes propriedades:

(D1) $\alpha \cdot(x+y)=\alpha \cdot x+\alpha \cdot y, \quad \forall \alpha \in \mathbb{K}$ e $\forall x, y \in X$,

(D2) $(\alpha+\beta) x=\alpha \cdot x+\beta \cdot x, \quad \forall \alpha, \beta \in \mathbb{K}$ e $\forall x \in X$.

Exemplo 1: O conjunto de polinômios

$$
\mathcal{P}(\mathbb{K})=\left\{p(x)=a_{n} x^{n}+\cdots+a_{1} x+a_{0} \mid a_{i} \in \mathbb{K} \text { e } n \geq 0\right\}
$$


é um espaço vetorial com as operações usuais de soma de polinômios e de multiplicação por escalar.

Definição 2: Sejam $U$ e $V$ espaços vetoriais sobre um corpo $\mathbb{K}$. Uma função $T: U \rightarrow V$ é uma transformação linear se

(1) $T\left(u_{1}+u_{2}\right)=T\left(u_{1}\right)+T\left(u_{2}\right)$, para todos $u_{1}, u_{2} \in U$,

(2) $T(\lambda u)=\lambda T(u)$, para todo $\lambda \in \mathbb{K}$ e $u \in U$.

Definição 3: Sejam $E, F$ espaços com produto interno e $T: E \rightarrow F$ uma aplicação. Uma aplicação $T^{*}: E \rightarrow F$ é adjunta de $T$, se satisfizer

$$
\langle T x, y\rangle=\left\langle x, T^{*} y\right\rangle \quad \forall x \in E, y \in F .
$$

No nosso trabalho, fizemos um estudo sobre um caso particular de operadores definido a seguir:

Definição 4: Sejam $E$ um espaço euclidiano e $T: E \rightarrow E$ um operador linear. Dizemos que $T$ é auto-adjunto se $T^{*}=T$.

Teorema 1 (Espectral): Sejam $X$ um espaço vetorial complexo de dimensão $n$ e $T: X \rightarrow X$ um operador linear com polinômio característico

$$
p(z)=\left(z-\lambda_{1}\right)^{s_{1}} \cdots\left(z-\lambda_{l}\right)^{s_{l}},
$$

em que os autovalores $\lambda_{i}$ são distintos, para $i=1, \cdots, l$.

Então existem subespaços $W_{1}, \ldots, W_{l}$ invariantes por $T$ (isto é, $T\left(W_{i}\right) \subset W_{i}$ ) tais que

$$
X=W_{1} \oplus \cdots \oplus W_{l} .
$$

Os espaços $W_{i}$ estão relacionados com autovetores do operador $T$.

Lema 1: Sejam $E$ um espaço euclidiano e $H: E \rightarrow E$ um operador auto-adjunto. Então

(i) $H$ possui apenas autovalores reais;

(ii) autovetores correspondentes a autovalores distintos são ortogonais.

Demonstração: (i) Supondo que $E$ seja um espaço complexo. Seja $x$ um autovetor associado ao autovalor $\lambda$ de $H$. Então,

$$
\lambda\langle x, x\rangle=\langle\lambda x, x\rangle=\langle H x, x\rangle=\langle x, H x\rangle=\langle x, \lambda x\rangle=\bar{\lambda}\langle x, x\rangle
$$

de modo que 


$$
(\lambda-\bar{\lambda})\langle x, x\rangle=0 .
$$

Assim, $\lambda=\bar{\lambda}$.

(ii) Sejam $x, y$ autovetores associados aos autovalores distintos $\lambda, \mu \in \mathbb{R}$.

Então

$$
\lambda\langle x, y\rangle=\langle\lambda x, y\rangle=\langle H x, y\rangle=\langle x, H y\rangle=\langle x, \mu y\rangle=\mu\langle x, y\rangle
$$

de modo que

$$
(\lambda-\mu)\langle x, y\rangle=0
$$

Como $\lambda \neq \mu$, temos que $\langle x, y\rangle=0$, então $x \perp y$.

Teorema 2 (Espectral dos Operadores Auto-adjuntos): Sejam $E$ um espaço euclidiano complexo e $H: E \rightarrow E$ um operador auto-adjunto. Então os autovetores de $H$ formam uma base ortogonal de $E$.

O Teorema 2 nos diz que um operador auto-adjunto é diagonalizável.

Lema 2: Seja $E$ um espaço euclidiano. Um operador auto-adjunto $H: E \rightarrow F$ é positivo e semidefinido se, e somente se, seus autovalores forem todos maiores do que ou iguais a zero. 0 operador $H$ é positivo definido se, e somente se, todos os seus autovalores forem positivos.

Teorema 3 (Raiz Quadrada): Sejam $E$ um espaço euclidiano e $H: E \rightarrow E$ um operador autoadjunto e positivo semidefinido. Então $H$ possui uma única raiz quadrada positiva semidefinida $P: E \rightarrow E$.

Demonstração: Consideremos a decomposição de $E$ como soma direta ortogonal de autoespaços de $E=E_{\lambda_{1}} \oplus \cdots \oplus E_{\lambda_{k}}$, em que $\lambda_{1}, \ldots, \lambda_{k}$ são autovalores distintos de $H$. Esta decomposição se deve ao Teorema 1. Se $x=x_{1}+\cdots+x_{k} \in E_{\lambda_{1}} \oplus \cdots \oplus E_{\lambda_{k^{\prime}}}$ então $H x=$ $\lambda_{1} x_{1}+\cdots+\lambda_{k} x_{k}$. Definimos $P_{x}=\sqrt{\lambda_{1}} x_{1}+\cdots+\sqrt{\lambda_{k}} x_{k}$. Temos que $P^{2} x=H x$, mostrando que $P$ é uma raiz quadrada de $H$ e pelo Lema 2 temos que $P$ é positivo e semidefinido.

Exemplo 2: Seja o operador auto-adjunto $T: \mathbb{C}^{2} \rightarrow \mathbb{C}^{2}$ definido por $T(z, w)=(2 z+$ $(1+i) w,(1+i) z+3 w)$.

A matriz desta transformação em relação à base canônica será

$$
[T]_{\text {can }}=\left[\begin{array}{cc}
2 & 1+i \\
1-i & 3
\end{array}\right] .
$$


Calculando os autovetores temos

$$
\begin{gathered}
{[T-x I]_{\text {can }}=\left[\begin{array}{cc}
2-x & 1+i \\
1-i & 3-x
\end{array}\right]} \\
p(x)=\operatorname{det}[T-x I]_{\text {can }}=(x-1)(x-4) .
\end{gathered}
$$

Assim, os autovalores são $x=1$ e $x=4$.

Para calcular os autovetores encontramos os vetores que satisfazem as relações $T(v)=1$. $v$ e $T(v)=4 \cdot v$. Os vetores $v_{1}=(1+i, 1)$ e $v_{2}=(1+i, 2)$ que, respectivamente, satisfazem as relações que precisávamos, assim, todos os múltiplos de $v_{1}$ satisfazem a primeira igualdade e todos os múltiplos de $v_{2}$ satisfazem a segunda igualdade.

Temos que $v_{1}$ e $v_{2}$ são associados a autovalores distintos, então são ortogonais entre si, e formam uma base ortogonal $\mathcal{B}=\left\{v_{1}, v_{2}\right\}$ para o espaço $\mathbb{C}^{2}$. Assim, temos a seguinte matriz de $T$ na base $\mathcal{B}$ :

$$
[T]_{\mathcal{B}}=\left[\begin{array}{ll}
1 & 0 \\
0 & 4
\end{array}\right]
$$

Extraindo a raiz quadrada dos autovalores, temos o operador

$$
[P]_{\mathcal{B}}=\left[\begin{array}{ll}
1 & 0 \\
0 & 2
\end{array}\right]
$$

tal que $[P]_{\mathcal{B}}^{2}=[T]_{\mathcal{B}}$, ou seja, o operador $P$ é uma raiz quadrada do operador $T$, e é único, segundo o Teorema apresentado.

\section{DISCUSSÃO}

Somente operadores não-negativos possuem raiz quadrada auto-adjunta, pois resulta imediatamente que da definição de $A^{*}$ que o quadrado de um operador auto-adjunto é nãonegativo. Entretanto, o quadrado de um operador de outro tipo pode ser um operador (autoadjunto) negativo. Por exemplo, a rotação de $90^{\circ}$ no plano tem quadrado igual à rotação de 180으, que é igual a $-I$. Além disso, um operador positivo pode ter uma raiz quadrada que não é autoadjunta. Por exemplo, o operador $A: \mathbb{R}^{2} \rightarrow \mathbb{R}^{2}$, definido por $A(x, y)=(2 x-y, 3 x-2 y)$ é uma raiz quadrada da identidade $I_{\mathbb{R}^{2}}$.

\section{CONCLUSÃO}

Neste trabalho, estudamos tópicos avançados de Álgebra Linear com enfoque no estudo de Operadores Auto-adjuntos. Com base na Diagonalização de Operadores Auto-Adjuntos e no Teorema Espectral pudemos notar que nos casos de Operadores Auto-adjuntos Positivos 
Semidefinidos existe uma única raiz quadrada semidefinida para um operador em espaços vetoriais Euclidianos.

O desenvolvimento do trabalho possibilitou o contato com técnicas importantes necessárias para o tratamento teórico empregado em Álgebra Linear e que são importantes para a continuidade do estudo de temas na área.

\section{REFERÊNCIAS}

1. BUENO, H. Álgebra Linear, Um Segundo Curso. 1.ed. Rio de Janeiro: SBM, 2006.

2. COELHO, F.; LOURENÇO, M. Um curso de Álgebra Linear. 2.ed. rev. e ampl. 2.reimp. São Paulo: Editora da Universidade de São Paulo, 2010.

3. LIMA, E. Álgebra Linear. 8.ed. Rio de Janeiro: IMPA 2011. 